\title{
REVIEW
}

\section{From a retrovirus infection of mice to a long noncoding RNA that induces proto-oncogene transcription and oncogenesis via an epigenetic transcription switch}

\author{
Alan Garen ${ }^{1,2,3}$
}

\begin{abstract}
Here I review the properties of the mouse retroelement VL30-1, which apparently derived from retrotranspostions of a founder VL30 retrovirus that infected the mouse germline after the mouse-human speciation. The VL30-1 gene is transcribed as a long noncoding RNA (IncRNA) with an essential host function in an epigenetic transcription switch (ETS) that regulates transcription of multiple genes, including proto-oncogenes that control cell proliferation and oncogenesis. The ETS involves the tumor suppressor protein PSF that has a DNA-binding domain (DBD) and two RNA-binding domains (RBDs). The DBD binds to promoters that have a DBD-binding sequence and switches off transcription, and the RBDs bind IncRNAs that have a RBD-binding sequence, releasing PSF and switching on transcription. VL30-1 IncRNA has two RBD-binding sequences, apparently acquired by mutations during retrotranspositions of the founder retrovirus, which drive proto-oncogene transcription and oncogenesis via the ETS. VL30-1 IncRNA is a seminal example of the key role of endogenous retroviruses (ERVs) and their retroelements in the evolution of transcription regulatory systems.
\end{abstract}

Signal Transduction and Targeted Therapy (2016) 1, 16007; doi:10.1038/sigtrans.2016.7; published online 13 May 2016

The operon model of gene regulation, a founding concept of molecular biology proposed by Jacob and Monod in 1961 based on their studies with Escherichia coli, ${ }^{1}$ focused attention on protein-coding genes as the fundamental functional component of all genomes, as affirmed in Monod's statement that 'anything found to be true for $E$. coli must also be true for the elephant.' Although it was known that mammalian genomes also contained DNA that did not encode any proteins, such DNA was usually called useless or selfish. ${ }^{2,3}$ The revelation from whole-genome sequencing that protein-coding genes comprise only a minuscule part of a mammalian genome, $\sim 2 \%$ of the human and mouse genomes, ${ }^{4-6}$ was a wake-up call to understand how most of the genomic DNA survived evolutionary selection for the fittest organisms. An associated revelation was that $\sim 8-10 \%$ of the human and mouse genomes consist of ERVs presumably derived from retroviral infections of the mammalian germlines. ${ }^{7-9}$ An ERV initially functions as a selfish DNA that integrates at multiple genomic sites via successive cycles of duplicative retrotansposition (DRT), involving integration, transcription, reverse transcription and integration at another genomic site, which must eventually be suppressed in order for the host to survive, while the ERV must acquire a beneficial host function to survive as a component of the host genome.

Here I discuss the remarkable properties of a mouse ERV called VL30-1, ${ }^{10}$ a member of the VL30 ERV family ${ }^{11}$ that probably originated from an infection of the mouse germline by a founder retrovirus after mouse-human speciation, as there are no VL30related sequences in the human genome. The mouse genome currently is estimated to contain 150-200 VL30-related sequences, ranging from a full-length $5-6-k b p$ VL30 gene that has the features of an ERV, notably $5^{\prime}$ and $3^{\prime}$ long terminal repeats (LTRs), to a single 'solo' LTR. ${ }^{11,12}$ In the full-length VL30 genes sequenced so far, including VL30-1, the internal DNA flanked by the LTRs contains multiple mutations, including stop codons in all three reading frames, which block translation of the retroviral proteins required for further DRT cycles. Although the DRT cycles are suppressed, at least some of the full-length VL30 genes, including VL30-1, are transcribed as a IncRNA with a poly-A tail and are exported to the cytoplasm.

The VL30-1 IncRNA was discovered in an experiment involving transfection of a human tumor cell by a retroviral vector produced in a mouse cell containing VL30-1 IncRNA, resulting in encapsulation of VL30-1 InCRNA in the retroviral particles and integration in the host genome as an ERV, which increased the metastatic potential of the host. ${ }^{10}$ Further studies showed that the increase in metastatic potential was caused by a novel mechanism of gene regulation involving the protein PSF $^{13}$ and a PSF-binding RNA. ${ }^{14-17}$ PSF contains a DBD and two homologous RBDs (RBD-1 and RBD-2; Figure 1). The DBD in PSF binds to the promoter of a gene containing a DBD-binding site and

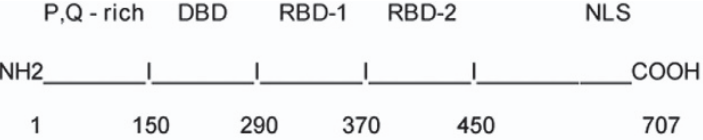

$$
\begin{aligned}
& \text { RBD1: } L-F-V-G-N-L \text { and } K-G-F-G-F-I-K-L \text {. } \\
& \text { RBD2: } L-S-V-R-N-L \text { and } T-G-K-G-I-V-E-F
\end{aligned}
$$

Figure 1. Organization of the PSF protein. The upper panel shows the DNA-binding domain (DBD) followed by the two RNA-binding domains (RBD-1 and RBD-2) and nuclear-localization signals (NLSs). ${ }^{13,19,24}$ The lower panel shows the RBD consensus residues in black and the non-consensus residues in red. ${ }^{19,25}$

\footnotetext{
${ }^{1}$ Department of Molecular Biophysics and Biochemistry, Yale University, New Haven, CT, USA; ${ }^{2}$ Sichuan University, Department of Obstetric and Gynecologic, West China Second University Hospital, Chengdu, China and ${ }^{3}$ State Key Laboratory of Biotherapy, West China Hospital, Chengdu, China.

Correspondence: A Garen (alan.garen@yale.edu)

Received 24 February 2016; revised 24 March 2016; accepted 28 March 2016
} 

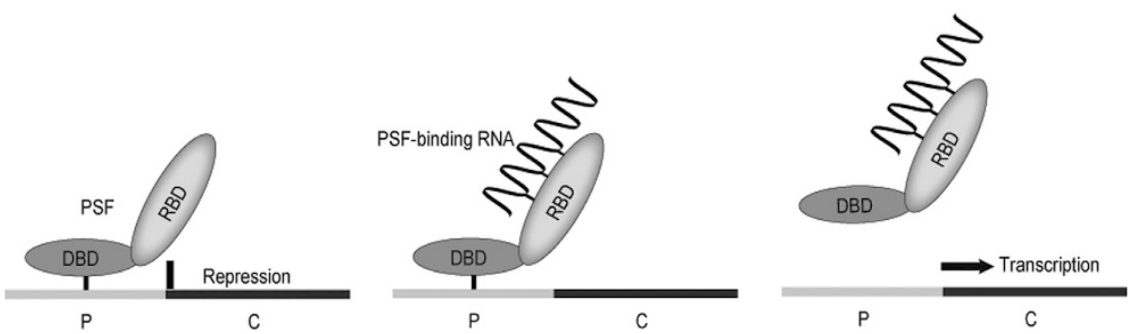

Figure 2. Regulation of transcription by PSF protein and PSF-binding RNA. The first diagram on the left shows the binding of the DBD in PSF to the promoter $(\mathrm{P})$ of a gene, causing repression of transcription of the coding region $(C)$. The second diagram in the center shows the binding of a RNA molecule to the RBD-1 and RBD-2 regions in PSF. The third diagram on the right shows the release of PSF from the promoter and initiation of transcription. ${ }^{16}$

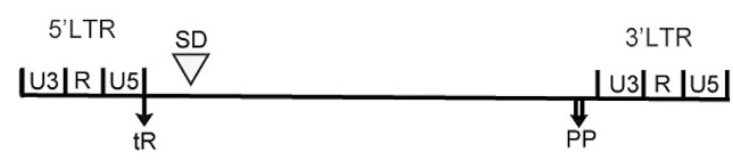

Figure 3. Map of VL30-1 IncRNA. The map shows the $5^{\prime}$ and $3^{\prime}$ LTRs, tR (tRNA primer binding site), SD (splice donor site) and PP (polypurine tract). ${ }^{10}$

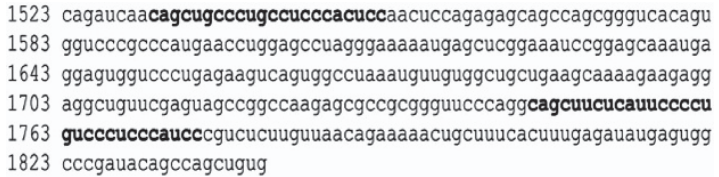
CAGCUG------CCCUG--CCUCCCACUCC CAGCUUCUCAUUCCCCUGUCCCUCCCA-UCC

Figure 4. PSF-binding sequences in VL30-1 IncRNA. ${ }^{14}$ The upper panel shows the two PSF-binding sequences (in bold) located in the region spanning nucleotides 1523-1841 of the full-length VL30-1 IncRNA, which contains 4939 nucleotides. The lower panel shows a comparison of the identical nucleotides in the two PSF-binding sequences (in black) and the non-identical nucleotides (in red).

represses transcription, ${ }^{14,18}$ and the RBD-1 and RBD-2 bind RNAs, usually a IncRNA, and reverse repression by PSF (Figure 2). ${ }^{14-17}$ This mechanism of gene regulation, which I term an ETS, regulates the transcription of multiple genes, including proto-oncogenes that control cell division and proliferation, and the P450scc gene that controls steroid synthesis. ${ }^{14-17}$ The PSF gene is strongly conserved between mice and humans, ${ }^{19}$ whereas the major PSF-binding RNAs differ yet retain the same function in the ETS. The major PSF-binding RNA in mice is VL30-1 IncRNA, which has the features of a LTR retroelement (Figure 3 ). VL30-1 IncRNA has an essential function in driving cell proliferation during mouse development, and it also has a deleterious function in inducing the oncogenic transformation of normal cells. $^{14-17}$ The PSF-binding sequences in VL30-1 IncRNA are localized in two short homologous regions, one with 22 nucleotides and another with 30 nucleotides (Figure 4), which probably were generated during the DRT cycles. Because VL30-1 IncRNA is exported to the cytoplasm after transcription (unpublished data), it must be imported back to the nucleus to bind to PSF; the import mechanism is not known.

The PSF gene probably existed in the mouse genome before the retrovirus infection that generated the VL30-1 gene. Although PSF protein is expressed during early development when cells proliferate, it does not function as a repressor until cells begin to differentiate and proliferation stops (unpublished data). Consequently, another PSF-binding IncRNA RNA, probably MALAT-1, ${ }^{20,21}$ was needed before VL30-1 IncRNA was available, to prevent binding of PSF to proto-oncogenes during early development. As VL30-1 IncRNA binds more effectively to PSF than MALAT-1 IncRNA (unpublished data), it could have co-opted the role of MALAT-1 IncRNA as the major PSF-binding RNA in the mouse ETS, providing an explanation for the surprising finding that MALAT-1 IncRNA is dispensable for mouse development and survival. ${ }^{22,23}$ I propose that the beneficial function of VL30-1 IncRNA, which was needed for its evolutionary survival in the mouse genome, was achieved in this way, providing a seminal example of the importance of retroviruses and their retroelement descendants in shaping the evolution of epigenetic systems for regulating gene transcription.

\section{COMPETING INTERESTS}

The author declares no conflict of interest.

\section{REFERENCES}

1 Jacob F, Monod J. Genetic regulatory mechanisms in the synthesis of proteins. J Mol Biol 1961; 3: 318-356.

2 Orgel LE, Crick FH. Selfish DNA: the ultimate parasite. Nature 1980; 284: 604-607.

3 Dawkins R. The Selfish Gene. Oxford University Press: Oxford, UK, 1976.

4 Lander ES, Linton LM, Birren B, Nusbaum C, Zody MC, Baldwin J et al. Initial sequencing and analysis of the human genome. Nature 2001; 409: 860-921.

5 Venter JC, Adams MD, Myers EW, Li PW, Mural RJ, Sutton GG et al. The sequence of the human genome. Science 2001; 291: 1304-1351.

6 Mouse Genome Sequencing Consortium, Waterston RH, Lindblad-Toh K, Birney E, Rogers J, Abril JF et al. Initial sequencing and comparative analysis of the mouse genome. Nature 2002; 420: 520-562.

7 Jern P, Coffin JM. Effects of retroviruses on host genome function. Annu Rev Genet 2008; 42: 709-732.

8 McCarthy EM, McDonald JF. Long terminal repeat retrotransposons of Mus musculus. Genome Biol 2004; 5: R14.

9 Stoye JP. Studies of endogenous retroviruses reveal a continuing evolutionary saga. Nat Rev Microbiol 2012; 10: 395-406.

10 Song X, Wang B, Bromberg M, Hu Z, Konigsberg W, Garen A. Retroviral-mediated transmission of a mouse VL30 RNA to human melanoma cells promotes metastasis in an immunodeficient mouse model. Proc Natl Acad Sci USA 2002; 99: 6269-6273.

11 French NS, Norton JD. Structure and functional properties of mouse VL30 retrotransposons. Biochim Biophys Acta 1997; 1352: 33-47.

12 Rotman G, Itin A, Keshet E. 'Solo' large terminal repeats (LTR) of an endogenous retrovirus-like gene family (VL30) in the mouse genome. Nucleic Acids Res 1984; 12: $2273-2282$.

13 Patton JG, Porro EB, Galceran J, Tempst P, Nadal-Ginard B. Cloning and characterization of PSF, a novel pre-mRNA splicing factor. Genes Dev 1993; 7: 393-406.

14 Song X, Sun Y, Garen A. Roles of PSF protein and VL30 RNA in reversible gene regulation. Proc Natl Acad Sci USA 2005; 102: 12189-12193.

15 Wang G, Cui Y, Zhang G, Garen A, Song X. Regulation of proto-oncogene transcription, cell proliferation, and tumorigenesis in mice by PSF protein and a VL30 noncoding RNA. Proc Natl Acad Sci USA 2009; 106: 16794-16798.

16 Garen A, Song X. Regulatory roles of tumor-suppressor proteins and noncoding RNA in cancer and normal cell functions. Int J Cancer 2008; 122: 1687-1689.

17 Song X, Sui A, Garen A. Binding of mouse VL30 retrotransposon RNA to PSF protein induces genes repressed by PSF: effects on steroidogenesis and oncogenesis. Proc Natl Acad Sci USA 2004; 101: 621-626. 
18 Urban RJ, Bodenburg Y, Kurosky A, Wood TG, Gasic S. Polypyrimidine tract-binding protein-associated splicing factor is a negative regulator of transcriptional activity of the porcine p450scc insulin-like growth factor response element. Mol Endocrinol 2000; 14: 774-782.

19 Dye BT, Patton JG. An RNA recognition motif (RRM) is required for the localization of PTB-associated splicing factor (PSF) to subnuclear speckles. Exp Cell Res 2001; 263: 131-144.

20 Li L, Feng T, Lian Y, Zhang G, Garen A, Song X. Role of human noncoding RNAs in the control of tumorigenesis. Proc Natl Acad Sci USA 2009; 106: 12956-12961.

21 Ji Q, Zhang L, Liu X, Zhou L, Wang W, Han Z et al. Long non-coding RNA MALAT1 promotes tumor growth and metastasis in colorectal cancer through binding to SFPQ and releasing oncogene PTBP2 from SFPQ/PTBP2 complex. Br J Cancer 2014; 111: 736-748.

22 Zhang B, Arun G, Mao YS, Lazar Z, Hung G, Bhattacharjee G et al. The IncRNA Malat1 is dispensable for mouse development but its transcription plays a cis-regulatory role in the adult. Cell Rep 2012; 2: 111-123.
23 Eißmann M, Gutschner T, Hämmerle M, Günther S, Caudron-Herger M, Groß M et al. Loss of the abundant nuclear non-coding RNA MALAT1 is compatible with life and development. RNA Biol 2012; 9: 1076-1087.

24 Dong X, Shylnova O, Challis JRG, Lye SJ. Identification and characterization of the protein-associated splicing factor as a negative co-regulator of the progesterone receptor. J Biol Chem 2005; 280: 13329-13340.

25 Maris $\mathrm{C}$, Dominguez $\mathrm{C}$, Allain $\mathrm{FH}$. The RNA recognition motif, a plastic RNA-binding platform to regulate post-transcriptional gene expression. FEBS J 2005; 272: 2118-2131.

(c) This work is licensed under a Creative Commons Attribution 4.0 International License. The images or other third party material in this article are included in the article's Creative Commons license, unless indicated otherwise in the credit line; if the material is not included under the Creative Commons license, users will need to obtain permission from the license holder to reproduce the material. To view a copy of this license, visit http://creativecommons.org/licenses/ by/4.0/ 